\title{
Empleo de instrumentos de evaluación comprensiva en Economía Aplicada
}

\section{The use of tools for comprehensive evaluation in Applied Economics}

José-Ignacio Antón.

Universidad de Salamanca.

janton@usal.es

Alexandra Jima-González.

Tecnológico de Antioquía, Institución Universitaria (Medellín, Colombia). alexandra.jima@tdea.edu.co

Miguel Paradela-López.

Tecnológico de Antioquía, Institución Universitaria (Medellín, Colombia). miguel.paradela@tdea.edu.co

Fernando Pinto Hernández. Universidad de Salamanca. fpinto@usal.es

\section{RESUMEN.}

El presente trabajo describe la implementación y resultados de una experiencia de innovación docente consistente en emplear herramientas de evaluación comprensiva en una asignatura de Economía Aplicada. El establecimiento de un sistema de evaluación que combine elementos de naturaleza prospectiva, formativa y sumativa responde a las necesidades del nuevo paradigma de enseñanza-aprendizaje basado en competencias preconizado por el Espacio Europeo de Educación Superior y los principales expertos en este ámbito. En particular, esta acción docente contempla la combinación de elementos de evaluación de carácter prospectivo (un test de nivel al comienzo de la asignatura), formativo (presentación de un examen tipo y realización de un test de repaso) y sumativo (instrumentos más convencionales que complementan al test de repaso) en la asignatura Economía del Estado del Bienestar. Para valorar esta experiencia docente, realizamos una encuesta ad hoc a los estudiantes y comparamos el rendimiento académico antes y después de la intervención en la asignatura y la satisfacción de los estudiantes con la actividad docente con el observado en otras materias similares. Los estudiantes mostraron un elevado nivel de satisfacción con la estrategia de evaluación comprensiva, cuya introducción, además, está correlacionada de forma positiva con el rendimiento académico y la satisfacción con la actividad docente, si bien resulta arriesgado establecer una relación causal. Los resultados del trabajo sugieren que combinar elementos de evaluación de distinta naturaleza (prospectiva, formativa y sumativa) resulta una acción eficiente (efectiva y con un coste reducido para el docente) para mejorar el rendimiento y la satisfacción con la actividad docente de los estudiantes.

\section{PALABRAS CLAVE.}

Evaluación comprensiva, evaluación prospectiva, evaluación formativa, evaluación sumativa, rendimiento académico, satisfacción con la actividad docente.

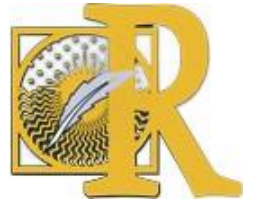




\section{ABSTRACT.}

This work describes the implementation and results of a teaching innovation consisting in employing tools for comprehensive evaluation in a course in Applied Economics. To resort to an evaluation system that combines prospective, formative and summative instruments fulfills the needs of the new paradigm of teaching and learning the European Space of Higher Education and the most qualified expert voices in this field preconize. Particularly, we introduce tools for prospective (a level test), formative (a sample exam and a review test) and summative assessment (some more conventional instruments complementing the review test) in a course on Economics of the Welfare State. In order to judge the impact of our teaching experience, we perform an ad hoc survey among students and we compare the students' academic performance before and after the introduction of these tools and the students' satisfaction with course with the one reported in other similar courses. The students of the course where we implement the innovation show a higher degree of satisfaction with the strategy of comprehensive evaluation and we observe a positive correlation between the use of these methods and academic performance and students' satisfaction, although it is risky to claim the existence of causal relationship. The results of our study suggest that the combination of assessment tools of a different nature (prospective, formative and summative) might mean an efficient strategy (effective and with a reasonable cost for the teaching staff) for improving the performance and the satisfaction with teaching of students.

\section{KEY WORDS.}

Comprehensive evaluation, prospective evaluation, formative evaluation, summative evaluation, academic performance, students' satisfaction with teaching.

\section{Introducción'.}

Las voces expertas más cualificadas y las principales agencias de evaluación de la calidad de la docencia universitaria subrayan la conveniencia de ofrecer un proceso de evaluación que abarque diferentes dimensiones. La adaptación de la enseñanza universitaria a las directrices del Espacio Europeo de Educación Superior (EEES) implica, entre otras cuestiones, el establecimiento de sistemas de evaluación centrados en las competencias, lo que demanda el empleo de herramientas que vayan más allá de instrumentos de naturaleza sumativa (Delgado, Borge, García \& Salomón, 2006; Cano, 2008; Fernández March, 2008, 2010; Cano et al., 2011; Agència per a la Qualitat del Sistema Universitari de Catalunya, 2009; Agencia Nacional de Evaluación de la Calidad y Acreditación, 2013).

En este sentido, la evaluación ha sido considerada no únicamente como un proceso sumativo de calificaciones, sino como un elemento pedagógico enfocado en el desarrollo de ciertas competencias: desde la capacidad para analizar y resolver problemáticas inherentes a la profesión (Bunk, 1994), hasta el desarrollo personal en contextos familiares o sociales (Stephenson \& Yorke, 1998). De acuerdo al trabajo desarrollado por Roe (2002), el proceso formativo del alumno no se limita a la incorporación sistemática de contenido, sino que también incluye habilidades prácticas o interpersonales. Por tanto, defiende un proceso de evaluación integral que tome en consideración que el alumno no sólo adquiere una serie de

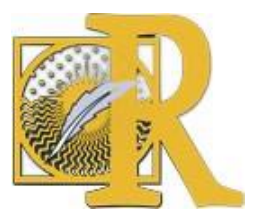


conocimientos durante su periodo universitario, sino que también experimenta un proceso de desarrollo personal y social.

El concepto de competencia ha sido objeto de enconado debate. Algunos autores e instituciones han aportado definiciones del mismo, destacando la propuesta de la norma UNE 66173 (Asociación Española de Normalización y Certificación, 2003), que define la competencia como "el conjunto de atributos personales y la aptitud demostrada para aplicar conocimientos y habilidades". Del mismo modo, Ríos Muñoz y Herrera Araya (2017, p. 1076) la definen como "saberes combinados que integran el ser, el saber hacer y el saber estar". No obstante, otros autores asumen la necesidad de construir diferentes concepciones (Tardif, 2003) o reconocen el carácter dinámico del concepto (Lasnier, 2000), lo que implica un grado de indeterminación en el mismo.

Por otro lado, numerosos pedagogos y académicos han señalado la mayor idoneidad de la educación comprensiva frente a un modelo clásico debido a las características de las sociedades contemporáneas. De acuerdo a Cano (2008), el contexto actual requiere la formación de ciudadanos capacitados para manejar de manera rápida y eficiente una gran cantidad de información, desarrollando una formación integral que les permita no sólo hacer frente a las problemáticas del momento, sino también adaptarse a nuevos contextos que puedan surgir en el futuro. En este sentido, la sociedad posmoderna implica un grado de indeterminación e inestabilidad para el que la formación clásica encuentra falencias: frente al conocimiento estático y "fotográfico", debe acudirse a una educación dinámica o "videográfica" (Tardif, 2006). La evaluación comprensiva se centra, en definitiva, no tanto en la adquisición de conocimientos, sino de unas habilidades que permitan obtener y analizar la información existente y utilizarla para adaptarse a los diferentes contextos en los que el alumno se encuentre (Álvarez, 2011).

En Europa, a pesar de que se ha reconocido la importancia de evaluar los resultados del aprendizaje mediante el desarrollo de competencias, hasta el momento el sistema europeo se ha centrado en la unificación curricular de los programas, buscando una homogeneización de las titulaciones que hiciera posible el reconocimiento recíproco de los mismos y, por ende, promoviendo la movilidad y el establecimiento de ciudadanos europeos en los países vecinos. Por ello, la evaluación ha tendido a ser relegada como un elemento secundario en la política educativa europea, lo que ha supuesto una desconexión entre los procesos de enseñanza y aprendizaje (Cano García, 2008). En este sentido, la práctica de la evaluación en la actualidad continúa teniendo un carácter tradicional, desligando la evaluación del proceso educativo - a través del de la retroalimentación y de la participación activa del alumno - para centrarlo mayoritariamente en la calificación (Ibarra \& Rodríguez, 2010). En este sentido, a pesar del avance en las últimas décadas, la práctica de la evaluación comprensiva continúa siendo minoritaria en el espacio europeo, lo que según Bolívar (2015) constituye un ejercicio pendiente en los diseños educativos de la región.

Más concretamente, en España, la práctica más tradicional y estándar de evaluación hasta hace poco tiempo consistía en el empleo de herramientas de valoración de los procesos de enseñanza aprendizaje (en el peor de los casos, meramente conocimientos adquiridos) (De Miguel et al., 2006). El Real Decreto 1393/2007 de 29 de octubre, si bien establece la necesidad de implementar un sistema de competencias basado en la evaluación comprensiva, se centra fundamentalmente en el acercamiento de los programas dentro del

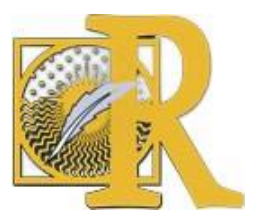


denominado Plan Bolonia. Esto ha supuesto que, si bien España ha logrado homologar sus titulaciones con las del resto de países europeos, continúa basándose fundamentalmente en sistemas de evaluación sumativa. Según Sevilla (2002), los motivos de este poco éxito ha sido la sobrevaloración de la escuela como factor social, la ineficacia de las reformas legislativas y la falta de apoyo docente a los procesos de cambio. No obstante, destacan esfuerzos para avanzar en el desarrollo de planes de evaluación comprensiva, como son el estudio sobre la evaluación no sumativa en la Universidad de Sevilla (González González, Arquero Montaño \& Hassall, 2014) o la propuesta de programas de formación del profesorado para incorporar herramientas de evaluación comprensiva en los docentes españoles (D’Angelo \& Rusinek. 2014).

Sin embargo, de acuerdo con la literatura especializada mencionada, la evaluación debe abarcar 3 dimensiones: diagnóstica o inicial, evaluativa y sumativa (Gargallo Castel, 2009). La finalidad de la evaluación diagnóstica, realizada al comienzo del curso, es proporcionar un punto de partido sólido para los procesos de enseñanza-aprendizaje que tendrán lugar a lo largo de cada una de las asignaturas propuestas. Estas evaluaciones tienen como finalidad que los docentes tomen conocimiento de cuáles es el nivel de aprendizaje y conocimiento de los estudiantes, principalmente, en relación a las competencias generales y específicas más relevantes en la asignatura.

Se pretende comprobar, por un lado, si los estudiantes poseen las competencias y conocimientos previos requeridos para el proceso concreto de enseñanza-aprendizaje que va a comenzar y, por otro, cuáles son sus conocimientos sobre el proceso que comienza. Por su parte, la evaluación formativa pretende proporcionar información al docente y al estudiante sobre el desarrollo de los procesos de enseñanza-aprendizaje antes de que se lleven a cabo algunas de las actividades de evaluación sumativa (en particular, los exámenes). Esta evaluación se orienta fundamentalmente a generar un proceso de retroalimentación, que permita a los estudiantes conocer con total precisión los criterios de evaluación y calificación y evaluar su propio nivel de aprendizaje, para, de esa forma, ayudar a aprender, fomentar un estudio inteligente y corregir errores a tiempo. Por último, la evaluación sumativa representaba la mayor parte de las acciones de evaluación en el entorno educativo previo al EEES. Consiste en aquellas herramientas que pretenden evaluar los procesos de enseñanza aprendizaje, transformándose normalmente en calificaciones comparables entre los estudiantes.

En tal contexto, el presente trabajo describe el diseño de un proceso de evaluación comprensiva, que abarca los tres ejes mencionados (diagnóstico o prospectivo, formativo y sumativo), y sus resultados en el contexto de una asignatura del área de Economía Aplicada en el Grado de Recursos Humanos y Relaciones Laborales impartido en la USAL durante el curso 2017-2018. 


\section{Material y métodos.}

El contexto de implementación de esta innovación docente correspondió a la asignatura Economía del Estado del Bienestar durante el curso 2017-2018. Se trata de una materia que se imparte dentro del Grado en Relaciones Laborales y Recursos Humanos, impartida en la Facultad de Ciencias Sociales. Esta asignatura, de carácter obligatorio, consta de 6 créditos (en términos de la nomenclatura del European Credit Transfer System, ECTS) y corresponde al $4^{\circ}$ curso $\left(2^{\circ}\right.$ semestre) de la titulación mencionada. La materia comprende los principales elementos de un curso introductorio en Economía Pública centrado en los programas de protección social y educación y destinado a alumnos que no pertenecen a la titulación de Economía o similares ${ }^{2}$. Esta materia presenta algunas peculiaridades que resulta pertinente señalar. En primer lugar, se trata de una materia a cargo del Departamento de Economía Aplicada dirigida a estudiantes cuya principal área de estudio es otra. Este elemento, que no es en absoluto infrecuente en nuestras universidades, resulta en la necesidad de llevar a cabo necesarias adaptaciones no solo para sortear dificultades del aprendizaje asociadas a conocimientos previos en la materia, sino, también, para lograr una implicación y motivación satisfactoria de los estudiantes. En segundo término, Economía del Estado del Bienestar se imparte dentro del último cuatrimestre del Grado, periodo durante el cual solo existen dos materias y que, por lo tanto, se reserva para la realización de las prácticas presenciales curriculares. Este hecho, sumado a que este Grado, en no pocas ocasiones, constituye la segunda titulación para personas que se encuentran integradas en el mercado laboral (por ejemplo, titulados en Derecho o Psicología) genera que la asistencia a clase sea, en términos generales, muy reducida, entre el 10 y el $30 \%$ dependiendo del horario. Este último hecho favoreció que tanto la implementación de algunos de los instrumentos de evaluación como los mecanismos para la recogida de información destinada a documentar esta acción docente se valga de herramientas de docencia en línea.

La asignatura se impartía a dos grupos, en horario de mañana (lunes de 9-11 horas y martes 9-10 horas) y tarde (lunes de 16-18 horas y martes de 16-17 horas), que contaban con 33 y 36 estudiantes, respectivamente. La afluencia en el grupo de mañana resulta, normalmente, sustancialmente más baja que por la tarde. La idea inicial para evaluar un proyecto pasaba por aleatorizar la intervención, implementando estos procesos en un único grupo, de forma que el otro podía servir como grupo de control. Esta idea inicial fue desechada por varios motivos: la existencia de contacto frecuente entre los dos grupos, el carácter no totalmente aleatorio de la configuración de los grupos asociado a problemas de compatibilización de la asistencia a las clases con actividades laborales o prácticas curriculares y la posible aparición de problemas con las autoridades académicas de la Facultad de Ciencias Sociales, que anteponen la homogeneidad formal de la docencia a otras consideraciones ${ }^{3}$. Dado que tuvo que descartarse una evaluación de carácter experimental, que hubiese permitido una estimación rigurosa del efecto causal de la intervención, se emplearon dos instrumentos alternativos, una encuesta ad hoc dirigida a los estudiantes para medir su grado de satisfacción con la asignatura y, específicamente, con la evaluación comprensiva, una análisis antes-después de los resultados académicos del curso 2017-2018 en relación a los observados al curso anterior (2016-2017) en la

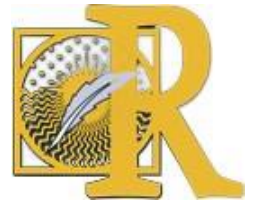


misma asignatura y una análisis comparativo entre los grados de satisfacción subjetiva de los estudiantes en la asignatura en relación con otras materias similares en titulaciones de Ciencias Sociales y Jurídicas.

Como se ha mencionado más arriba, la evaluación comprensiva incluye 3 tipos de herramientas: prospectivas o diagnósticas, formativas y sumativas. En todos los casos, los instrumentos utilizados tenían en consideración las peculiaridades y dificultades derivadas del contexto que se ha descrito en líneas anteriores.

En primer lugar, la evaluación diagnóstica, por su propia naturaleza, debe llevarse a cabo durante las primeras semanas del curso, con la mayor premura posible, para modificar adecuadamente las estrategias de enseñanza-aprendizaje, logrando una total coherencia entre los medios, resultados esperados y el punto de partida de los procesos de enseñanzaaprendizaje. En este caso, los docentes elaboraron un test de respuesta múltiple de 32 preguntas, con 4 respuestas posibles, de las cuales solo una de ellas era correcta. Las respuestas incorrectas implicaban una penalización de 1/3. Las preguntas pretendían calibrar los conocimientos de los estudiantes en materia de Economía en general y Economía Laboral en particular. El Grado en Relaciones Laborales y Recursos Humanos cuenta con una asignatura introductoria de Economía (6 ECTS, formación básica), una materia en Economía de los Recursos Humanos (6 ECTS, formación básica, consistente en una Economía Laboral desde la perspectiva de la empresa) y un curso en Economía Laboral (4 ECTS, obligatorio). El test hacía énfasis en medir la comprensión de los estudiantes de conceptos básicos, como conceptos básicos relacionados con la medición de la actividad económica, el funcionamiento del mercado de trabajo, los principales fundamentos de las políticas macroeconómicas, las elasticidades de oferta y demanda o los tipos de imposición y sus efectos, entre otros. El cuestionario se elaboró a través del software Hot Potatoes (2019) y se puso a disposición de los estudiantes a través de la plataforma Moodle de la USAL. Los estudiantes contaban debían responder al test durante las primeras semanas del curso y el resultado no tenía implicación alguna sobre la calificación final de la asignatura. El objetivo del ejercicio era recabar información sobre el grado de adquisición de competencias específicas de los estudiantes relacionados con el área de Economía Aplicada en cursos anteriores, con la finalidad de realizar adaptaciones pertinentes en la asignatura que facilitasen el proceso de enseñanza aprendizaje. Así, por ejemplo, en varias unidades didácticas se repasan de forma previa a los contenidos evaluables algunos conceptos básicos que son necesarios que sirven de recordatorio y que son necesarios para comprender los temas que se abordan. Del mismo modo, esta información permite dedicar más tiempo a aquellas áreas en las cuales los estudiantes presenten mayores carencias o dificultades o, incluso en algunos casos extremos, rediseñar unidades didácticas o reconsiderar la dificultad de algunos contenidos o tareas. Este test, que tuvo carácter voluntario, fue realizado por alrededor de un tercio de los estudiantes.

La evaluación formativa se implementó a través de dos instrumentos distintos: un ejemplo de examen y un test de repaso. La evaluación formativa consistió en proporcionar un examen de muestra similar al que tendrían que enfrentarse los estudiantes al final de la asignatura. Dicho ejercicio constaba de preguntas abiertas de respuesta larga en la que los estudiantes cuentan con cierta posibilidad de elección ${ }^{4}$. Asimismo, los estudiantes contaban con la posibilidad de remitir al profesor por correo electrónico la contestación a una de las

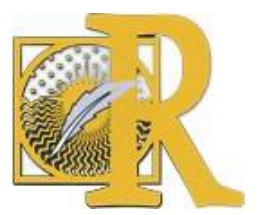


preguntas y recibir un feedback (una calificación explicada de la pregunta junto con consejos pertinentes de cara al examen real). Aproximadamente un $5 \%$ de los estudiantes hizo uso de esta posibilidad. El segundo elemento de evaluación formativa empleado consistía en un test final, consistente en una prueba de respuesta múltiple de 25 preguntas (con 3 posibles respuestas y una penalización de 1/2). Este test, elaborado e implementado con las mismas herramientas que la prueba diagnóstica, se encontraba orientado al repaso y cubría los principales contenidos de las unidades didácticas del programa. Se planteó en las últimas semanas del curso y el límite para su realización era el en el que se realizaba el examen escrito final en convocatoria ordinaria. Suponía el $10 \%$ de la calificación final y, por ende, tenía naturaleza obligatoria y representaba un instrumento de evaluación tanto formativa como sumativa.

El conjunto de actividades de evaluación viene completado por una serie de herramientas de valoración de naturaleza sumativa, entre las cuales destacan, por encima de otras actividades de carácter voluntario, un examen de respuesta múltiple sobre una serie de 9 lecturas obligatorias (20\% de la nota), la entrega de 4 comentarios de texto propuestos por el profesor (20\% de la nota) y el mencionado examen escrito de preguntas abiertas de respuesta larga (50\% de la calificación final) ${ }^{5}$.

Como se mencionó con anterioridad, en relación a los instrumentos empleados para valorar el éxito de la actividad, la primera de las herramientas utilizadas consistió en una encuesta elaborada y realizada a través de la plataforma Moodle de la USAL. Consistió en 5 preguntas de escala Likert a las que los estudiantes debían responder indicando su nivel de satisfacción de 1 (mínimo) a 5 (máximo). La escala utilizada coincide con la empleada por la USAL a en las encuestas de satisfacción de los estudiantes con la actividad docente. Las preguntas específicas se presentan a continuación:

1. ¿Cómo valorarías tu experiencia en la asignatura Economía del Estado del Bienestar?

2. En este curso, se han tratado de incluir tres tipos evaluación distinta: prospectiva (test inicial), evaluativa (preguntas de examen tipo que puedes consultar al profesor) y sumativa (test final y exámenes al final de la asignatura). ¿Cómo valorarías la utilización de este sistema en la asignatura Economía del Estado del Bienestar?

3. ¿Te parece una buena idea la utilización del test de conocimientos iniciales por parte del profesor (para conocer el nivel de los alumnos, adaptar la asignatura, etc.)?

4. ¿Te parece una buena idea que el profesor suministre un modelo de examen para que los

5. ¿Te parece una buena idea que el profesor haga un test final en Moodle que sirve como repaso?

El segundo instrumento empleado en este trabajo viene dado por la comparación del rendimiento académico obtenido en la asignatura en el curso 2017-2018 y el correspondiente al curso anterior (2016-2017) y la comparación entre el grado de satisfacción con la actividad docente en las encuestas realizadas por Unidad de Evaluación de la Calidad (UEC) de la USAL durante el curso 2017-2018 en la materia en la que se implementó esta iniciativa de innovación docente $y$, por un lado, otras asignaturas relacionadas con el área de Economía Aplicada que se imparten titulaciones de Ciencias 
Sociales y, por otro, con las materias Hacienda Pública II y Hacienda Pública que forman parte del Grado en Economía y del Grado en Derecho, respectivamente, y comparten una proporción sustancial de sus contenidos con Economía del Estado del Bienestar ${ }^{6}$. En relación al rendimiento académico, el análisis explora las diferencias en varios estadísticos descriptivos (media, mediana, percentil 20, percentil 80 y la ratio entre el percentil 80 y el percentil 20, una medida muy simple de dispersión) haciendo uso de métodos de inferencia estadística (contrastes de hipótesis para ver si las diferencias en estos estadísticos en 2016-2017 y 2017-2018 son significativas estadísticamente). En relación a las asignaturas cuya satisfacción con la actividad docente exploramos y comparados con que es objeto de la intervención descrita en este trabajo, consideramos las siguientes materias: Economía, Estructura Económica Española, Economía de los Recursos Humanos y Economía Laboral (Grado en Relaciones Laborales y Recursos Humanos); Economía Política, Introducción a la Economía, Economía del Estado del Bienestar y Mercado de Trabajo y Educación (Grado en Sociología) Hacienda Pública. Para este segundo ejercicio, se seleccionamos únicamente el promedio de los 11 ítems que componen la encuesta (y que es el principal elemento empleado por la UEC para la parte de la evaluación de la actividad docente basada en estas encuestas) y las 2 preguntas con mayor interés para el tipo de intervención implementada ("Los métodos de evaluación se corresponden con el desarrollo docente de la materia" [ítem P10] y "Mi grado de satisfacción general con el/la profesor/a ha sido bueno" [ítem P11]).

Resulta de indudable relevancia señalar las importantes limitaciones que de las que adolece este segundo bloque de métodos para detectar el efecto causal de la intervención. En particular, la comparación entre cursos académicos está lastrada por la existencia de otras variables que cambian a lo largo del tiempo y que pueden estar correlacionadas con la intervención, como, por ejemplo, las diferencias de rendimiento entre las distintas cohortes de estudiantes. Además, parte de las intervenciones de forman parte de la evaluación comprensiva ya se habían implementado con anterioridad y hubo otros elementos de la organización de la asignatura que se modificaron y que podrían afectar a los resultados académicos ${ }^{7}$. En relación a la comparación con la satisfacción subjetiva reportada por los estudiantes en otras asignaturas no cabe duda de que, a pesar de que se trata del mismo curso académico, las diferencias observadas en esta variable entre la asignatura Economía del Estado del Bienestar y estas materias puede encontrarse asociada multitud de factores que, desafortunadamente, no es posible tener en cuenta en nuestro análisis (dificultad de la materia, profesor de la asignatura, etc.). En ambos casos, por lo tanto, es más conveniente hablar de la existencia de correlación simple que de causalidad, lo cual invita a tomar los resultados con mucha precaución. Al menos, pretendemos establecer si no existe una correlación negativa entre el uso de sistemas de evaluación comprensiva y otras variables relevantes; en otras palabras, si, al menos parece encontrarse en vigor el principio de "no perjudicar", ampliamente empleado en Medicina.

Por último, cabe mencionar que los datos se procesaron con el software Stata 15.1 (StataCorp, 2017). 


\section{Resultados.}

Los resultados de la aplicación de las herramientas de recogida de información y análisis de la misma se presentan en la Figura 1 y las Tablas 1 y 2 . La Figura 1 recoge los resultados de la encuesta de satisfacción docente realiza a los estudiantes, mostrando la distribución de las respuestas de acuerdo a la escala 1-5 empleada y el promedio de las mismas. Los resultados que refleja el gráfico de lo misma apuntan a unos niveles elevados de valoración del empleo de una pluralidad de instrumentos de evaluación. Quizás de modo poco sorprendente, destaca especialmente, la importancia otorgada por el alumnado a contar con un modelo de examen, que hace la evaluación más transparente y elimina incertidumbre. La precaución más relevante a la hora de interpretar estos resultados está vinculada a la existencia de un posible sesgo de selección (solo responde a la encuesta aproximadamente el $30 \%$ de los estudiantes) y los asociados a la propia subjetividad de este tipo de cuestionarios.

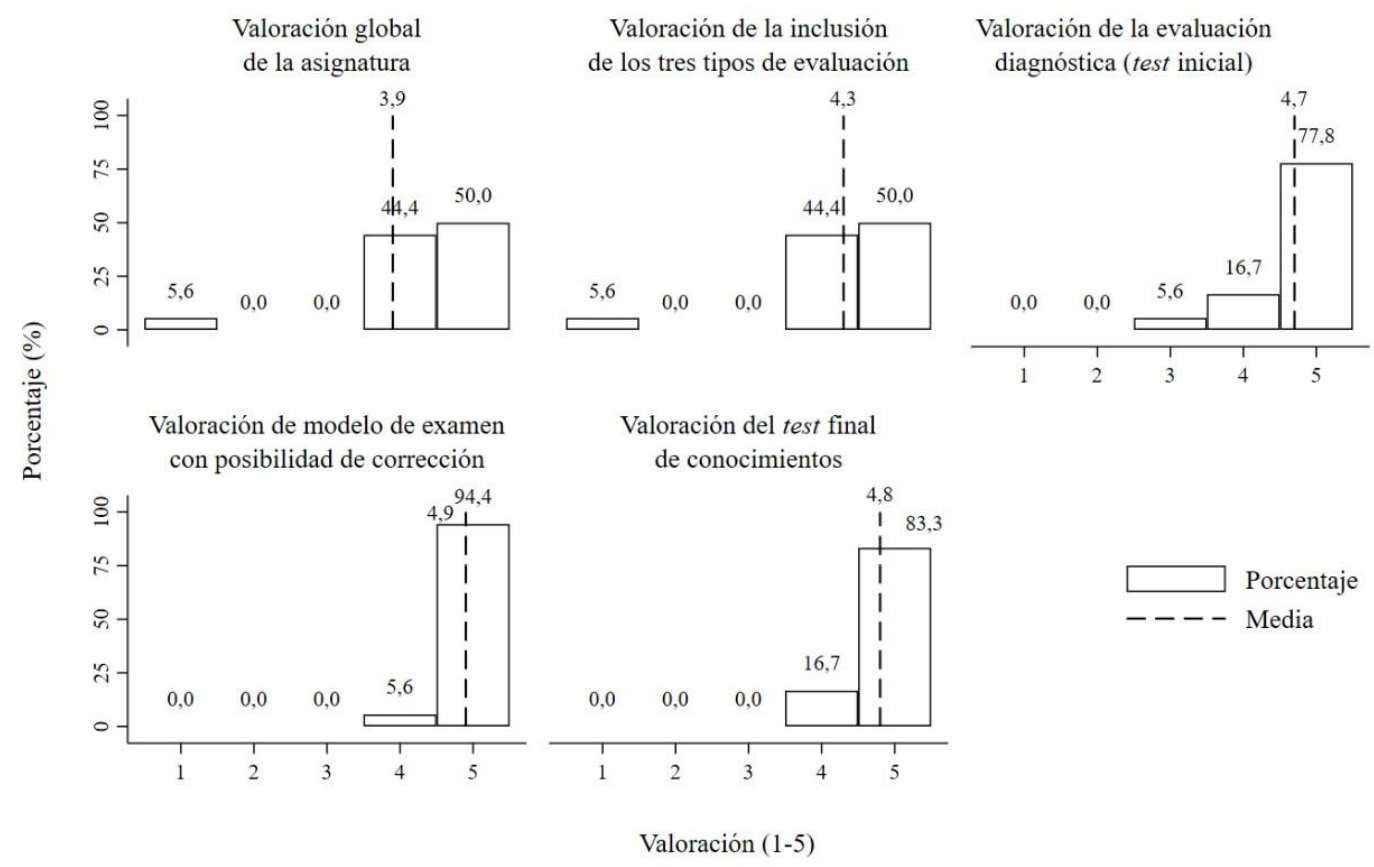

Figura 1. Valoración subjetiva de los estudiantes acerca de las estrategias de evaluación comprensiva en la asignatura "Economía del Estado del Bienestar" (curso 2017-2018).

Nota: N. de estudiantes matriculados: 69. Participación en la encuesta: 26,9\%.

Fuente: Elaboración propia a partir de la encuesta realizada a los estudiantes.

En segundo término, se presentan los resultados relativos a la evolución de las calificaciones entre el curso 2016-2017 y 2017-2018 (Tabla 2). El análisis de las diferencias entre los estadísticos descriptivos en ambos periodos sugiere un incremento de la calificación promedio, mediana y del percentil 20. Esto resulta, asimismo, en una reducción de la ratio entre el percentil 80 y 20 , esto es, de la dispersión entre las calificaciones más

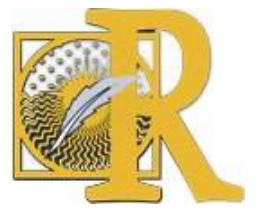


altas y más bajas. Como se señalo con anterioridad, la única evidencia que cabe extraer de estos resultados es el de la existencia de una correlación positiva y debe evitarse obtener conclusiones de carácter causal entre la presencia de la intervención y el rendimiento académico.

\begin{tabular}{|c|c|c|c|}
\hline & Curso 2016-2017 (1) & Curso 2017-2018 (2) & Diferencia $[(2)-(1)]$ \\
\hline \multirow[t]{2}{*}{ Promedio } & 7,072 & 8,075 & $1,003^{\star \star *}$ \\
\hline & $(0,137)$ & $(0,198)$ & $(0,241)$ \\
\hline \multirow[t]{2}{*}{ Mediana } & 7,000 & 8,300 & 1,300 \\
\hline & $(0,275)$ & $(0,275)$ & $(0,394)$ \\
\hline \multirow[t]{2}{*}{ Percentil 20} & 5,300 & 7,100 & $1,800^{* * *}$ \\
\hline & $(0,200)$ & $(0,200)$ & $(0,292)$ \\
\hline \multirow[t]{2}{*}{ Percentil 80} & 8,500 & 9,000 & 0,500 \\
\hline & $(0,325)$ & $(0,075)$ & $(0,340)$ \\
\hline \multirow[t]{2}{*}{ Percentil 80/percentil 20} & 1,604 & 1,268 & $0,336)^{\star \star \star \star}$ \\
\hline & $(0,082)$ & $(0,038)$ & $(0,044)$ \\
\hline 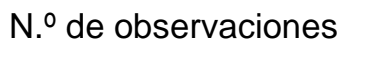 & 61 & 69 & \\
\hline
\end{tabular}

Tabla 1. Pruebas de igualdad entre varios estadísticos descriptivos del rendimiento académico en la asignatura "Economía del Estado del Bienestar" durante los cursos 2016-2017 y 2017-2018.

Notas: ${ }^{* *}$ significativo a un nivel del $1 \%$; ${ }^{*}$ significativo a un nivel del $5 \%$; ${ }^{*}$ significativo a un nivel del $10 \%$. Errores estándar entre paréntesis. Fuente: Elaboración propia a partir de las calificaciones de los estudiantes.

Por último, este apartado procede a analizar los resultados de las encuestas de satisfacción con la actividad docente de la asignatura en relación a otras con la que comparte rasgos, ya sea en contenidos o en el hecho de que se trata de asignaturas de Economía Aplicada dirigidas a estudiantes de grado en otras Ciencias Sociales (Tabla 3). De la exploración de estos resultados podemos destacar tres elementos de interés. El primero de ellos hace referencia a que, en la pregunta relativa a los métodos de evaluación, si bien no recoge con exactitud la intervención que aquí estamos realizando, la asignatura objeto de evaluación comprensiva (Economía del Estado del Bienestar en el Grado en Relaciones Laborales y Recursos Humanos) obtiene la mayor puntuación de todas las consideradas con excepción de una (Estructura Económica Española). En segundo término, la satisfacción global con el profesor y el promedio de ítems de la encuesta es superior en la asignatura en la que se implementó la evaluación comprensiva que en el resto. Por último, la materia que se benefició de la acción docente sale particularmente bien parada cuando establecemos la comparación con la asignatura Economía del Estado del Bienestar del Grado en Sociología.

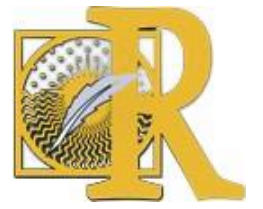


Estas asignaturas comparten en gran medida contenidos y competencias, si bien en el Grado en Sociología esta materia goza de carácter voluntario y consta de 4 ECTS. Como en el caso anterior, deben extremarse las precauciones antes de realizar cualquier interpretación causal de los resultados, ya que estos podrían estar guiados por otro tipo de factores que no están siendo tenidos en cuenta en el análisis y que quedan fuera de los objetivos del trabajo. No obstante, la evidencia presentada sí parece sugerir que el es poco probable que no se respete el principio de "no perjudicar" con la implementación de proceso de evaluación comprensiva.

"Los métodos de evaluación se corresponden con el desarrollo docente de la materia" (ítem P10)
"Mi grado de satisfacción general Promedio de los 11 con el/la profesor/a ítems de la encuesta ha sido bueno" (ítem (P1-P11) P11)

Grado en Relaciones Laborales y

Recursos Humanos

\begin{tabular}{|c|c|c|c|}
\hline Economía del Estado del Bienestar & 4,00 & 4,36 & 4,21 \\
\hline Economía & 3,92 & 3,25 & 3,44 \\
\hline Estructura Económica Española & 4,14 & 3,88 & 3,89 \\
\hline Economía Laboral & 2,35 & 2,16 & 2,59 \\
\hline $\begin{array}{l}\text { Economía de los Recursos Humanos } \\
\text { cado en Sociología }\end{array}$ & 3,62 & 3,68 & 3,59 \\
\hline Economía Política & 3,78 & 4,00 & 3,93 \\
\hline Introducción a la Economía & 4,04 & 4,28 & 4,10 \\
\hline Economía del Estado del Bienestar & 2,50 & 3,40 & 3,19 \\
\hline $\begin{array}{l}\text { Mercado de Trabajo y Educación } \\
\text { ado en Trabajo Social }\end{array}$ & 3,75 & 3,47 & 3,55 \\
\hline Economía & 3,96 & 3,52 & 3,62 \\
\hline Grado en Economía & & & \\
\hline $\begin{array}{l}\text { Hacienda Pública I } \\
\text { ado en Derecho }\end{array}$ & 3,72 & 3,52 & 3,51 \\
\hline Hacienda Pública & 3,45 & 3,17 & 3,33 \\
\hline
\end{tabular}

Tabla 2. Comparación de los resultados de encuestas de satisfacción del estudiante con la actividad docente del profesorado en distintas asignaturas en el curso 2017-2018.

Fuente: Elaboración propia a partir de UEC (2019a, 2019b, 2019c, 2019d, 2019e).

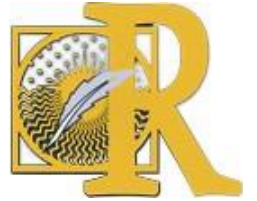




\section{Discusión.}

Este trabajo ha descrito una experiencia de innovación docente consistente en introducir métodos de evaluación comprensiva, combinando herramientas de naturaleza prospectiva, formativa y sumativa, en una asignatura del área de Economía Aplicada y apoyada en el empleo de herramientas de docencia en línea. Esta estrategia, consistente en aplicar elementos de evaluación de diferente naturaleza y que responde a las necesidades de adaptación de los sistemas de evaluación por competencias asociado al EEES y que preconizan las voces expertas en la materia y principales agencias de evaluación educativa, recibe una valoración muy positiva de los estudiantes, expresada a través de encuestas realizadas por los autores. Asimismo, el empleo de este tipo de instrumentos está correlacionada positivamente con la satisfacción de los estudiantes con la actividad docente y el rendimiento académico (incrementando su media y reduciendo su dispersión), aunque no resulta prudente realizar una interpretación causal de los resultados dada la más que probable influencia de terceros factores que no han podido incluirse en el análisis. Cabe reseñar que esta intervención no demanda un elevado esfuerzo adicional por parte del profesorado, de forma que, dada la correlación positiva con los indicadores de rendimiento y satisfacción, puede considerarse como una acción de innovación docente relativamente eficiente.

Por último, cabe realizar una serie de reflexiones para futuras investigaciones y mejora del funcionamiento de estos métodos. En primer lugar, aunque la evidencia presentada en el trabajo es sugerente, resulta inevitable subrayar la conveniencia de contar con evaluaciones del efecto de estos procedimientos de carácter experimental o cuasiexperimental que contengan un mayor rigor metodológico y permitan evitar los posibles sesgos de los resultados que se han discutido en el artículo. Asimismo, una posible vía para mejorar la evaluación formativa podría ser establecer la obligatoriedad del contestar a una de las preguntas del ejemplo de examen y enviarla al profesor, en lugar de permitir que esta actividad fuese voluntaria. Por supuesto, adoptar esta estrategia puede incrementar de forma notable el volumen de trabajo y es un aspecto que merece una reflexión más profunda. Además, para acrecentar la transparencia de los criterios de evaluación, esta actividad encontraría un importante aliado en la elaboración de una rúbrica para la valoración de las respuestas los estudiantes. Por último, resultaría relevante determinar el impacto diferenciado de cada uno elementos de innovación (esto es, los instrumentos de evaluación prospectiva o los de naturaleza formativa) en los procesos de enseñanzaaprendizaje. Las respuestas de los estudiantes indican, de forma preliminar, que son los relacionados con evaluación formativa (posiblemente, porque acrecientan la certidumbre sobre el sistema de evaluación y los criterios de corrección) aquellos que potencialmente redundan en mayores efectos positivos. 


\section{Referencias bibliográficas.}

- Agencia Nacional de Evaluación de la Calidad y Acreditación (2013). Guía de apoyo para la redacción, puesta en práctica y evaluación de los resultados del aprendizaje. Madrid: Agencia Nacional de Evaluación de la Calidad y Acreditación.

- Agència per a la Qualitat del Sistema Universitari de Catalunya (2009). Guía para la evaluación de competencias en el área de Ciencias Sociales. Barcelona: Agència per a la Qualitat del Sistema Universitari de Catalunya.

- Álvarez, M. M. (2011). Perfil del docente en el enfoque basado en competencias. Revista Electrónica Educare, 15(1), 99-107.

- Asociación Española de Normalización (2003). UNE 66173 IN: los recursos humanos en un sistema de gestión de la calidad: gestión de las competencias. Recuperado de: https://www.aenor.com/normas-y-libros/buscador-de-normas/une/?c=N0029960

- Bolívar, A. (2015). The Comprehensive School in Spain: A Review of its Development Cycle and Crises. European Educational Research Journal, 14(3-4), 347-363.

- Bunk, G. (1994). La transmisión de las competencias en la formación y perfeccionamiento profesionales de la RFA. Revista europea de formación profesional, $1,8-14$.

- Cano García, M. (2008). La evaluación por competencias en la educación superior. Profesorado. Revista de Currículum y Formación de Profesorado, 12(3), 1-16.

- Cano, E., Barrios, R., Cabrera, N., Delgado, A. M., Fabregat, J., Fernández, ... Valero, M. (2011). Buenas prácticas en la evaluación de competencias. Cinco casos de educación superior. Barcelona: Laertes Educación.

- D’Angelo, E. \& Rusinek, G. (2014). La evaluación de un programa de formación permanente del profesorado en España: metodología y diseño. Revista iberoamericana en educación, 64, 55-71.

- De Miguel, M., Alfaro, I. J., Apodaca, J. M., Arias, J. M., García, E., Lobato, C. \& Pérez, A. (2006). Metodologías de enseñanza y aprendizaje para el desarrollo de competencias. Madrid: Alianza Editorial.

- Delgado, A. M., Borge, R., García, J., Oliver, R. \& Salomón, L. (2006). Competencias y diseño de la evaluación continua y final en el Espacio Europeo de Educación Superior. Una experiencia desde el Derecho y la Ciencia Política. Barcelona: J. M. Bosch.

- Fernández March, A. (2008). La evaluación de los aprendizajes en la universidad: nuevos enfoques. Recuperado de: https://web.ua.es/es/ice/documentos/recursos/materiales/evaprendizajes.pdf.

- Fernández March, A. (2010). La evaluación orientada al aprendizaje en un modelo de formación por competencias en la educación universitaria. REDU: Revista de Docencia Universitaria, 8(1), 11-34.

- Gargallo Castel, A. (2009). Aproximación al estudio de la evaluación en la universidad: un análisis exploratorio con alumnos semipresenciales. Contextos educativos: Revista de educación, 12, 77-93.

- González González, J. M.; Arquero Montaño, J. L. \& Hassall, T. (2014). Consolidación de la formación por competencias en la universidad española: Estudio de un caso. Educación XX1, 17(2), 145-168. 
- Hot Potatoes (7.0.0.19) [Software]. Recuperado de: https://hotpot.uvic.ca.

- Ibarra, M.S. \& Rodríguez, G. (2010). Aproximación al discurso dominante sobre la evaluación del aprendizaje en la universidad. Revista de Educación, 351, 385-407.

- Lasnier, F. (2000). Réussir la formation par compétences. Montreal: Guérin.

- Roe, R.A. (2002). What Makes a Competent Psychologist? European Psychologist, 7(3), 192-202.

- Sevilla Merino, D. (2003). La educación comprensiva en España: paradoja, retórica y limitaciones. Revista de educación, 330, 35-57.

- StataCorp (2017). Stata Statistical Software: Release 15 (version 15.1). College Station, Texas: StataCorp Limited Liability Company.

- Stephenson, J. \& Yorke, M. (1998). Creating the Conditions for the Development of Capability. En J. Stephenson \& M. Yorke (Eds.), Capability and Quality in Higher Education (193-225). Londres: Kogan Page Editorial.

- Tardif, J. (2003). Développer un programme par compétences: de l'intention à la mise en oeuvre. Pédagogie collégiale, 16(3), 36-44.

- Tardif, J. (2006). L'évaluation des compétences. Documenter le parcours de développement. Montreal: Chenelière Éducation.

- Unidad de Evaluación de la Calidad de la Universidad de Salamanca (2019a). Informe de resultados. Curso 2017-2018. Encuesta de satisfacción del estudiante con la actividad docente del profesorado. Grado en Derecho. Facultad de Derecho. Recuperado

de: https://www.usal.es/files/grados/indicadores/2019/W G223 SATAD1718 Derecho.pdf

- Unidad de Evaluación de la Calidad de la Universidad de Salamanca (2019b). Informe de resultados. Curso 2017-2018. Encuesta de satisfacción del estudiante con la actividad docente del profesorado. Grado en Economía. Facultad de Economía y Empresa. Recuperado https://www.usal.es/files/grados/indicadores/2019/W G237 SATAD1718 Economia.pdf.

- Unidad de Evaluación de la Calidad de la Universidad de Salamanca (2019c). Informe de resultados. Curso 2017-2018. Encuesta de satisfacción del estudiante con la actividad docente del profesorado. Grado en Relaciones Laborales y Recursos Humanos. Facultad de Ciencias Sociales. Recuperado de: https://www.usal.es/files/grados/indicadores/2019/W G245 SATAD1718 RRLLyRRHH SA.pdf

- Unidad de Evaluación de la Calidad de la Universidad de Salamanca (2019d). Informe de resultados. Curso 2017-2018. Encuesta de satisfacción del estudiante con la actividad docente del profesorado. Grado en Sociología. Facultad de Ciencias Sociales. Recuperado de: https://www.usal.es/files/grados/indicadores/2019/W G270 SATAD1718 Sociologia.pdf 
- Unidad de Evaluación de la Calidad de la Universidad de Salamanca (2019e). Informe de resultados. Curso 2017-2018. Encuesta de satisfacción del estudiante con la actividad docente del profesorado. Grado en Trabajo Social. Facultad de Ciencias Sociales. Recuperado https://www.usal.es/files/grados/indicadores/2019/W G209 SATAD1718 TrabajoSocial. pdf

- Unidad de Evaluación de la Calidad de la Universidad de Salamanca (2018). Guía metodológica. Encuestas docentes. Recuperado de: https://drive.google.com/file/d/1NxCmGHrAqdD9cltiTM2kQhD4JF6qdK2v/view

$\overline{1}$ El presente trabajo se basa en los resultados del proyecto de innovación docente ID2017/109 Diseño e implementación de procesos de evaluación comprensiva desarrollado en la Universidad de Salamanca (USAL) y propuestas recogidas en el proyecto docente e investigador de Autor para el concurso de acceso a la plaza de Profesor Titular de Universidad G059B/D15911 en la USAL. Una versión previa del mismo fue presentada en las XV Jornadas sobre Docencia de Economía Aplicada (8 de marzo de 2019, Madrid, España). Los autores agradecen las sugerencias de dos evaluadores anónimos que contribuyeron a mejorar de forma sustancial una versión previa de este trabajo. Los posibles errores son naturalmente atribuíbles a los autores.

${ }^{2}$ Todos los materiales de la asignatura, incluyendo los instrumentos empleados para la evaluación, se han puesto a disposición de cualquier interesado a través de la iniciativa Open Course Ware de la USAL (http://ocw.usal.es/ciencias-sociales-1/economia-del-estado-del-bienestar).

${ }^{3}$ El contacto entre estudiantes de ambos grupos restaría validez a la aleatorización si, por ejemplo, el grupo de control accede a los materiales de la evaluación formativa propuestos para el grupo de tratamiento. Además, en algunos casos en los que la Secretaría de la Facultad no concedía oficialmente el cambio de grupo y el alumno, el profesor permitía a los alumnos acudir en el horario más conveniente.

${ }^{4}$ Sobre las ventajas e inconvenientes de este tipo de preguntas puede consultarse, por ejemplo, Fernández March (2008).

${ }^{5}$ En el curso $2017-2018$ se propusieron 2 actividades voluntarias: la asistencia a la presentación de un libro relacionado con el sistema de pensiones en la propia universidad y el visionado de una película. En ambos casos, los estudiantes debían responder a una serie de preguntas planteadas por el profesor con posteridad destinadas a los asistentes. Cada una de las actividades permitía incrementar la calificación final en 0,25 puntos adicionales.

${ }^{6}$ Para acceder a la metodología y cuestionario detallado de la encuesta puede acudirse a UEC (2018).

${ }^{7}$ Así, por ejemplo, el examen de muestra ya se proporcionaba en cursos anteriores, pero sin enfatizar mucho su relevancia y sin que se proporcionase feedback a los estudiantes. Asimismo, la evaluación de las lecturas descansaba sobre una serie de comentarios de texto en 2016-2017, mientras que en 2017-2018 eran objeto de un examen específico. De esta forma, aunque el profesor era el mismo en ambos cursos, existen elementos que hacen que los resultados hayan de ser tomados con precaución.

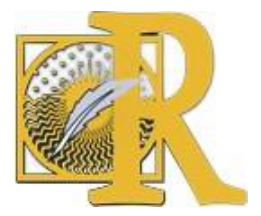

Fecha de recepción: 11-04-2019 Fecha de aceptación: 21-05-2019 